Faculty of Science

Faculty Publications

This is a post-print version of the following article:

Regularization by External Variables

Jeffrey, M., Glendinning, P., Edwards, R., Webber, S., \& Bossoline, E.

2017

The final publication is available at:

https://doi.org/10.1007/978-3-319-55642-0 4

Citation for this paper:

Jeffrey, M., Glendinning, P., Edwards, R., Webber, S., \& Bossoline, E. (2017)

Regularization by External Variables. In: Colombo A., Jeffrey M., Lázaro J., Olm

J. (eds) Extended Abstracts Spring 2016. Trends in Mathematics, vol 8.

Birkhäuser, Cham. https://doi.org/10.1007/978-3-319-55642-0 4 
Jeffrey, M., Glendinning, P., Edwards, R., Webber, S., \& Bossoline, E. (2017). Regularization by External Variables. In Extended Abstracts Spring 2016: Nonsmooth Dynamics (pp. 19-24). (Trends in Mathematics; Vol. 8). Birkhäuser Basel. https://doi.org/10.1007/978-3319-55642-0_4

Peer reviewed version

Link to published version (if available):

10.1007/978-3-319-55642-0_4

Link to publication record in Explore Bristol Research

PDF-document

This is the author accepted manuscript (AAM). The final published version (version of record) is available online via Springer at https://link.springer.com/chapter/10.1007\%2F978-3-319-55642-0_4 . Please refer to any applicable terms of use of the publisher.

\section{University of Bristol - Explore Bristol Research}

General rights

This document is made available in accordance with publisher policies. Please cite only the published version using the reference above. Full terms of use are available:

http://www.bristol.ac.uk/red/research-policy/pure/user-guides/ebr-terms/ 


\title{
Regularization by External Variables
}

\author{
E. Bossolini ${ }^{1}$, R. Edwards ${ }^{2}$, P. A. Glendinning ${ }^{3}$, M. R. Jeffrey ${ }^{4}$, S. Webber ${ }^{4}$ \\ 1. Department of Applied Mathematics and Computer Science, Technical University of Denmark, \\ Kongens Lyngby 2800, DK, ebos@dtu.dk \\ 2. Department of Mathematics and Statistics, University of Victoria, Victoria, BC, V8W 2Y2, Canada, \\ edwards@uvic.ca \\ 3. School of Mathematics, University of Manchester, Oxford Road, Manchester M13 9PL, UK, \\ p.a.glendinning@manchester.ac.uk \\ 4. Department of Engineering Mathematics, University of Bristol, Bristol BS8 1UB, UK, \\ mike.jeffrey@bristol.ac.uk
}

Regularization was a big topic at the 2016 CRM Intensive Research Program on Advances in Nonsmooth Dynamics. There are many open questions concerning well known kinds of regularization (e.g. by smoothing or hysteresis). Here we propose a framework for an alternative and important kind of regularization: by external variables.

\section{Shadowing in one variable}

Begin with a one-dimensional dynamical system

$$
\dot{x}=-\lambda+x b(x ; \lambda) \quad \text { where } \quad \lambda=\operatorname{sign}(x) .
$$

with the sign function being \pm 1 for $x \gtrless 0$ and having the set value $(-1,+1)$ for $x=0$. This has an attracting fixed point on the discontinuity, where $\dot{x}=-\lambda$.

Define a switch-shadowing system

$$
\dot{x}=-\lambda+x b(x ; \lambda), \quad \dot{y}=(x-y) / \gamma, \quad \text { where } \lambda=\operatorname{sign}(y),
$$

or a state-shadowing system

$$
\dot{x}=-\lambda+y b(y ; \lambda), \quad \dot{y}=(x-y) / \gamma, \quad \text { where } \quad \lambda=\operatorname{sign}(x),
$$

where $\gamma>0$ is small and $y$ is an external variable, representing some extra stage in the switching process, such that each shadow system relaxes to (11) as $y \rightarrow x$. So $y$ tends to $x$ like $e^{-t / \gamma}$ (for small $\gamma$ where we can treat $x$ as slow varying), i.e. $y$ shadows $x$.

We restrict attention to the neighbourhood of the equilibrium at $x=y=\lambda=0$ in each system. In the switch-shadowing system the switching surface becomes $y=0$, and sliding no longer occurs because solutions all cross the surface (because the $y$ component does not switch) - the surface is 'transparent' in some nomenclature. In the stateshadowing system the switching surface remains sliding.

We will analyze these using switching layer methods (see [1] and next section).

For the switch-shadowing system on $y=0$ the switching layer system is

$$
\dot{x}=-\lambda+x b(x ; \lambda), \quad \varepsilon \dot{\lambda}=x / \gamma, \quad \text { for } \quad \lambda \in(-1,+1), \quad \varepsilon \rightarrow 0,
$$

and the Jacobian of the equilibrium is

$$
\left(\begin{array}{ll}
\frac{\partial \dot{x}}{\partial x} & \frac{\partial \dot{x}}{\partial \dot{x}} \\
\frac{\partial \varepsilon \dot{\lambda}}{\partial x} & \frac{\partial \varepsilon \dot{\lambda}}{\partial \varepsilon \lambda}
\end{array}\right)=\left(\begin{array}{cc}
b & -1 / \varepsilon \\
1 / \gamma & 0
\end{array}\right)
$$


with eigenvalues $\left(b(0,0) \pm i \sqrt{4-b^{2} \gamma \varepsilon}\right) / 2 \sqrt{\gamma \varepsilon} \rightarrow \frac{1}{2} b(0,0) \pm i \infty$ as $\varepsilon \rightarrow 0$. Outside the switching surface the dynamics spirals in as a 'fused focus' towards $x=y=0$, but once there, in the $x$ - $\lambda$ dynamics the attractivity depends on the sign of $b(0,0)$. In particular if $b(0,0)>0$ then the sliding equilibrium will become unstable, and a limit cycle will be formed inside the switching layer $(x, \lambda) \in \mathbb{R} \times(-1,+1)$.

For the state-shadowing system on $x=0$ the switching layer system is

$$
\varepsilon \dot{\lambda}=-\lambda+y b(y ; \lambda), \quad \dot{y}=-y / \gamma, \quad \text { where } \lambda \in(-1,+1),
$$

and the Jacobian of the equilibrium is

$$
\frac{\partial(\varepsilon \dot{\lambda}, \dot{y})}{\partial(\varepsilon \lambda, y)}=\left(\begin{array}{cc}
-1 / \varepsilon & b(0 ; 0) \\
0 & -1 / \gamma
\end{array}\right)
$$

with eigenvalues $-1 / \gamma$ and $-1 / \varepsilon \rightarrow-\infty$. In this case the equilibrium of the shadow system remains an attractor.
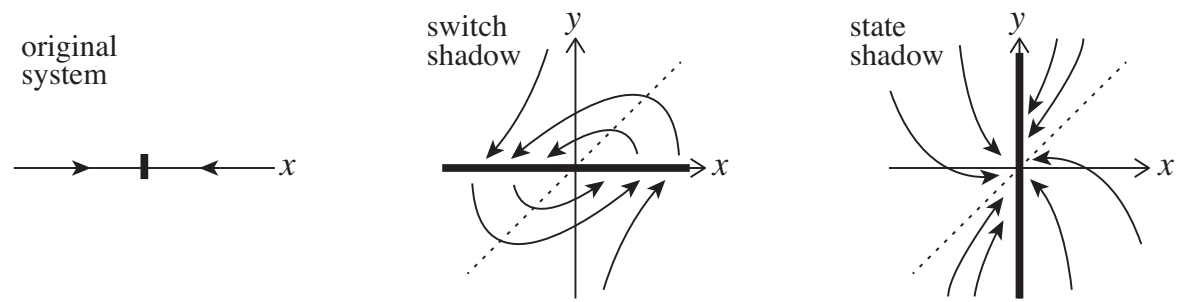

FigURE 1 . The original system and its two shadow regularizations.

\section{Shadowing in $n$ variables}

Now take a multivariable state $\mathbf{x}=\left(x_{1}, \ldots, x_{n}\right)$, and assume there is one switch for every coordinate (this can be generalized later). So we have switching functions $h_{1}, . ., . h_{n}$, and switching multipliers $\boldsymbol{\lambda}=\left(\lambda_{1}, \ldots, \lambda_{n}\right)$ where $\lambda_{i} \in[-1,+1]$, such that $\lambda_{i}=\operatorname{sign} h_{i}$ for $h_{i} \neq 0$ and $\lambda_{i} \in(-1,+1)$ for $h_{i}=0$. Letting $\mathbf{f}$ be a smooth function of $\mathbf{x}$ and $\boldsymbol{\lambda}$, the system

$$
\dot{\mathbf{x}}=\mathbf{f}(\mathbf{x} ; \boldsymbol{\lambda}) \quad \text { where } \quad \lambda_{i}=\operatorname{sign} h_{i}
$$

is smooth except at the thresholds $\Sigma_{i}=\left\{\mathbf{x} \in \mathbb{R}^{n}: h_{i}=0\right\}$.

In the piecewise smooth setting we assume each $h_{i}$ is a regular function of $\mathbf{x}$, some $h_{i}=h_{i}(\mathbf{x})$. When $h_{i}=0$ for some $i$, we blow up the switching surface $h_{i}=0$ into a switching layer $\lambda_{i} \in(-1,+1)$, with dynamics given by $\varepsilon_{i} \dot{\lambda}_{i}=\mathbf{f}(\mathbf{x} ; \boldsymbol{\lambda}) \cdot \nabla h_{i}$ for $\varepsilon_{i} \rightarrow 0$.

Take coordinates in which $h_{i}=x_{i}$ for $i=1, \ldots, n$. When $\mathbf{x}$ lies on the intersection of all $n$ switching thresholds $x_{1}=x_{2}=\ldots=x_{n}=0$, we study the dynamics in the codimension $n$ switching layer $\left(\lambda_{1}, \ldots, \lambda_{n}\right) \in(-1,+1)^{n}$ given by

$$
\underline{\underline{\varepsilon}} \dot{\boldsymbol{\lambda}}=\mathbf{f}(\mathbf{0} ; \boldsymbol{\lambda}), \quad|\underline{\underline{\varepsilon}}| \rightarrow 0,
$$

where $\underline{\underline{\varepsilon}}$ denotes the diagonal matrix with entries $\varepsilon_{1}, \ldots, \varepsilon_{n}$, or in components $\varepsilon_{i} \dot{\lambda}_{i}=$ $f_{i}\left(\mathbf{0} ; \lambda_{1}, \ldots, \lambda_{n}\right)$ for $i=1, \ldots, n$. Sliding modes are equilibria of the fast system. We assume these lie at $\mathbf{x}=\boldsymbol{\lambda}=0$, and are stable, which means

$$
\frac{\partial \underline{\underline{\varepsilon}} \cdot \dot{\lambda}}{\partial \underline{\underline{\varepsilon}} \cdot \boldsymbol{\lambda}}=\underline{\underline{\varepsilon}}^{-1} \cdot \frac{\partial \mathbf{f}}{\partial \boldsymbol{\lambda}} \text { has eigenvalues with negative real part at }(\mathbf{0} ; \mathbf{0}) .
$$

Define a switch-shadowing system

$$
\dot{\mathbf{x}}=\mathbf{f}(\mathbf{x} ; \boldsymbol{\lambda}), \quad \dot{\mathbf{y}}=(\mathbf{x}-\mathbf{y}) / \gamma, \quad \text { where } \quad \lambda_{i}=\operatorname{sign}\left(y_{i}\right),
$$


or a state-shadowing system

$$
\dot{\mathbf{x}}=\mathbf{f}(\mathbf{y} ; \boldsymbol{\lambda}), \quad \dot{\mathbf{y}}=(\mathbf{x}-\mathbf{y}) / \gamma, \quad \text { where } \quad \lambda_{i}=\operatorname{sign}\left(x_{i}\right),
$$

where $\gamma>0$ is small (we could choose different $\gamma_{i}$ for each component of $\mathbf{y}$ ), and $\mathbf{y}$ is an $n$-dimensional external variable. As before, both tend to (8) as $\mathbf{y}$ shadows $\mathbf{x}$.

Each has an equilibrium at $\mathbf{x}=\mathbf{y}=\boldsymbol{\lambda}=0$. For the switch-shadowing system on $\mathbf{y}=0$ the switching layer system is

$$
\dot{\mathbf{x}}=\mathbf{f}(\mathbf{x} ; \boldsymbol{\lambda}), \quad \varepsilon \dot{\boldsymbol{\lambda}}=\mathbf{x} / \gamma, \quad \text { for } \quad \boldsymbol{\lambda} \in(-1,+1)^{n},
$$

and the Jacobian of the equilibrium is

$$
\left(\begin{array}{cc}
\frac{\partial \dot{\mathbf{x}}}{\partial \mathbf{x}} & \frac{\partial \dot{\mathbf{x}}}{\partial \underline{\underline{\varepsilon} \cdot \lambda}} \\
\frac{\partial \underline{\underline{\underline{\varepsilon}} \cdot \dot{\boldsymbol{\lambda}}}}{\partial \mathbf{x}} & \frac{\partial \underline{\underline{\underline{\varepsilon}} \cdot \dot{\lambda}}}{\partial \underline{\underline{\varepsilon}} \cdot \boldsymbol{\lambda}}
\end{array}\right)=\left(\begin{array}{cc}
\frac{\partial \mathbf{f}(0 ; 0)}{\partial \mathbf{x}} & \underline{\underline{\varepsilon}}^{-1} \cdot \frac{\partial \mathbf{f}(0 ; 0)}{\partial \boldsymbol{\lambda}} \\
\underline{\underline{1}} / \gamma & 0
\end{array}\right)
$$

where $\underline{\underline{1}}$ is the $n \times n$ identity matrix. The stability of the term $\underline{\underline{\varepsilon}}^{-1} \cdot \frac{\partial \mathbf{f}}{\partial \boldsymbol{\lambda}}$ from (10) does not guarantee stability of the shadow equilibrium, which will depend crucially on $\frac{\partial \mathbf{f}(0 ; 0)}{\partial \mathbf{x}}$.

For the state-shadowing system on $x=0$ the switching layer system is

$$
\varepsilon \dot{\boldsymbol{\lambda}}=\mathbf{f}(\mathbf{y} ; \boldsymbol{\lambda}), \quad \dot{\mathbf{y}}=-\mathbf{y} / \gamma, \quad \text { where } \quad \lambda \in(-1,+1),
$$

and the Jacobian of the equilibrium is

$$
\frac{\partial(\underline{\underline{\varepsilon} \cdot \dot{\boldsymbol{\lambda}}, \dot{\mathbf{y}})}}{\partial(\underline{\underline{\varepsilon}} \cdot \boldsymbol{\lambda}, \mathbf{y})}=\left(\begin{array}{cc}
\underline{\underline{\varepsilon}}^{-1} \cdot \frac{\partial \mathbf{f}(0 ; 0)}{\partial \boldsymbol{\lambda}} & \frac{\partial \mathbf{f}(0 ; 0)}{\partial \mathbf{y}} \\
0 & -\underline{\underline{1}} / \gamma
\end{array}\right)
$$

In this case it seems likely that the equilibrium of the shadow system remains an attractor, the stability of the term $\underline{\varepsilon}^{-1} \cdot \frac{\partial \mathbf{f}}{\partial \boldsymbol{\lambda}}$ from (10) and the term $-\underline{\underline{1}} / \gamma$ playing the crucial role.

\section{Examples}

The following examples motivated the shadow regularizations proposed above.

- Genetic Regulatory Networks: a typical gene network protein-only model gives the dynamics of the concentration $x_{i}$ of the protein product of a gene $i$, for $i=1, \ldots, n$, as

$$
\dot{x}_{i}=B_{i}\left(Z_{1}, \ldots, Z_{n}\right)-\alpha_{i} x_{i}, \quad Z_{i}=\operatorname{step}\left(x_{i}-\theta_{i}\right),
$$

where $\alpha_{i}, \theta_{i}>0$. In [3] this is extended to include the intermediary role of mRNA. Instead we make $x_{i}$ the concentration of the $i^{\text {th }}$ mRNA molecule, and $y_{i}$ the protein product concentration for gene $i$, then the proposed model is

$$
\dot{x}_{i}=B_{i}\left(Z_{1}, \ldots, Z_{n}\right)-\alpha_{i} x_{i}, \quad \dot{y}_{i}=\kappa_{i} x_{i}-\beta_{i} y_{i}, \quad Z_{i}=\operatorname{step}\left(y_{i}-\theta_{i}\right),
$$

with $\alpha_{i}, \beta_{i}, \kappa_{i}, \theta_{i}>0$.

- Time delay: assume a system modelled by $\dot{x}=f(x ; \lambda)$ with $\lambda=\operatorname{sign}(x)$ actually switches not exactly when a solution $x(t)$ lies at $x(t)=0$, but when $x(t-\tau)$ with a time delay $\tau$. We can define a delayed variable $y(t)=x(t-\tau)$, or let

$$
\dot{x}=f(x ; \lambda), \quad \dot{y}=(x-y) / \tau, \quad \text { where } \quad \lambda=\operatorname{sign}(y) .
$$

- Plankton: a predator-prey system discussed in [4] for predator population $x_{3}$ and prey populations $x_{1}, x_{2}$, is

$$
\left.\begin{array}{l}
\dot{x}_{2}=\left\{r_{2}-x_{3}(1-\mu)\right\} x_{2} \\
\dot{x}_{3}=\left\{q_{1} x_{1} \mu+q_{2} x_{2}(1-\mu)-m\right\} x_{3}
\end{array}\right\} \quad \text { where } \quad \mu=\operatorname{step}\left(x_{1}-a x_{2}\right),
$$


in terms of constants $r_{1}, r_{2}, q_{1}, q_{2}, m, a$. This assumes the consumption of prey is proportional to their population $x_{1}$ or $x_{2}$. If instead consumption is proportional to a variable $y_{1}$ or $y_{2}$, which tends towards the population, we have

$$
\left.\begin{array}{l}
\dot{x}_{1}=r_{1} x_{1}-x_{3} y_{1} \mu \\
\dot{x}_{2}=r_{2} x_{2}-x_{3} y_{2}(1-\mu) \\
\dot{x}_{3}=\left\{q_{1} y_{1} \mu+q_{2} y_{2}(1-\mu)-m\right\} x_{3} \\
\dot{y}_{1}=\left(x_{1}-y_{1}\right) / \gamma_{1} \\
\dot{y}_{2}=\left(x_{2}-y_{2}\right) / \gamma_{2}
\end{array}\right\} \quad \text { where } \mu=\operatorname{step}\left(x_{1}-a x_{2}\right) \text {. }
$$

- Electronic sensors: a typical form for a piecewise affine control system is

$$
\dot{\mathbf{x}}=\underline{\underline{A}} \mathbf{x}+\mathbf{b} u \quad \text { where } \quad u=\operatorname{step}\left(x_{1}-\theta\right),
$$

in terms of a constant matrix $A$ and vector $\mathbf{b}$ describing electronic components. In $[2$ it is noted that, although a control system implements control on the state $\mathbf{x}$, it does so by measuring not $\mathbf{x}$ itself, but a sensor value $\mathbf{y}$, hence a more faithful model is

$$
\dot{\mathbf{x}}=\underline{\underline{A}} \mathbf{x}+\mathbf{b} u, \quad \dot{\mathbf{y}}=(\mathbf{x}-\mathbf{y}) . \underline{\underline{\kappa}}, \quad \text { where } \quad u=\operatorname{step}\left(y_{1}-\theta\right),
$$

for some diagonal matrix $\underline{\underline{\kappa}}$.

\section{A united form}

We can express both the switch and state shadow regularizations together by writing

$$
\dot{\mathbf{x}}=\mathbf{f}\left(\mathbf{s}_{\mu}(\mathbf{x}, \mathbf{y}) ; \boldsymbol{\lambda}\right), \quad \dot{\mathbf{y}}=(\mathbf{x}-\mathbf{y}) / \gamma, \quad \text { where } \quad \lambda_{i}=\operatorname{sign}\left(S_{\mu}\left(x_{i}, y_{i}\right)\right),
$$

for vector and scalar shadow functions $\mathbf{s}_{\mu}(\mathbf{x}, \mathbf{y})$ and $S_{\mu}(x, y)$ which satisfy $\mathbf{s}_{\mu}(\mathbf{x}, \mathbf{x})=\mathbf{x}$ and $S_{\mu}(x, x)=x$, for example $\mathbf{s}_{\mu}(\mathbf{x}, \mathbf{y})=\mu \mathbf{x}+(1-\mu) \mathbf{y}$ and $S_{\mu}(x, y)=\mu x+(1-\mu) y$. The switch-shadowing and state-shadowing systems are obtained at the extremes for $\mu=1$ and $\mu=0$ respectively. In the most general case we could consider $\gamma$ to be a (contracting) matrix, and/or a function of $\mathbf{x}$ and $\mathbf{y}$.

It will be interesting to study in the future how the stability of equilibria is affected under such regularizations in general, and the implications this has for the structural stability of piecewise smooth systems.

A final but important note must be made if the switching layer expression $\varepsilon \dot{\lambda}=\ldots$ is derived as the approximation to a smooth system (as in e.g. GRN models [3]). Then the $\varepsilon$ on the lefthand side of this expression is actually a function of $\lambda$, which makes the vanishing entries of the Jacobians from $\frac{\partial \varepsilon \dot{\lambda}}{\partial \varepsilon \lambda}$ become nonzero, and while we expect this not to qualitatively affect the result as $\varepsilon \rightarrow 0$, further study is required.

\section{References}

[1] P. Glendinning, M. R. Jeffrey (2016) An Introduction to Piecewise Smooth Dynamics. Advanced Courses in Mathematics - CRM Barcelona (Springer)

[2] G. Kafanas (2016) Sensor effects in sliding mode control of power conversion cells. Trends in Mathematics: Research Perspectives CRM Barcelona (Springer)

[3] R. Edwards, A. Machina, G. McGregor, and P. van den Driessche (2015) A modelling framework for gene regulatory networks including transcription and translation. Bull. Math. Biol., 77:953-983

[4] S. Piltz (2016) Smoothing a piecewise-smooth: An example from plankton population dynamics. Trends in Mathematics: Research Perspectives CRM Barcelona (Springer) 
Abstract: Regularization was a big topic at the 2016 CRM Intensive Research Program on Advances in Nonsmooth Dynamics. There are many open questions concerning well known kinds of regularization (e.g. by smoothing or hysteresis). Here we propose a framework for an alternative and important kind of regularization, by external variables that shadow either the state or the switch of the original system. The shadow systems are derived from and inspired by various applications in electronic control, predator-prey preference, time delay, and genetic regulation. 